\title{
ON THE IDENTIFICATION OF EIGENSTRAIN SOURCES OF WELDING RESIDUAL STRESS IN BEAD-ON-PLATE INCONEL 740H SPECIMENS
}

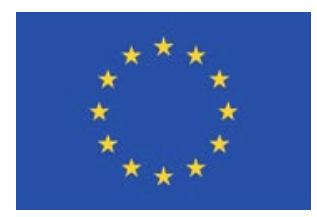 \\ Fatih Uzun ${ }^{1,2, *}$, Alexander M Korsunsky ${ }^{1,3}$ \\ ${ }^{1}$ MBLEM, Department of Engineering Science, The University of Oxford \\ ²fatihuzun@me.com, fatih.uzun@eng.ox.ac.uk \\ 3alexander.korsunsky@eng.ox.ac.uk, *corresponding author
}

\begin{abstract}
Authors’ Accepted Manuscript
Published version in International Journal of Mechanical Sciences

Volume 145, 2018, Pages 231-245
\end{abstract}

https://doi.org/10.1016/j.ijmecsci.2018.07.007 


\section{ABSTRACT}

The main source of welding residual stress is identified by investigating the distribution of permanent plastic strains in as-welded and post-weld heat treated specimens of Inconel $740 \mathrm{H}$. For this purpose, inverse eigenstrain problem is solved using experimental displacement data obtained by high precision coordinate measuring machine measurements from the surface of transversal wire-cut of electric discharge machining. A new multi-component iterative inverse eigenstrain model is developed and, in total, three different inverse eigenstrain models are analysed to get a high-quality fit with experimental data and have a reasonable prediction of residual stresses in the whole body of nonuniform bead-on-plate specimen design. In order to determine permanent plastic strains, which are the main source of welding residual stress, eigenstrain distribution size is analysed in terms of mean squared error using three models. The distribution size that provides the best fit between reconstructed displacements and the coordinate measuring machine measurements of deplanation on the surface of cut is determined. Multi-component iterative eigenstrain reconstruction model validated itself by providing good agreement with experimentally measured displacement and residual stress profiles, and this model is used to predict residual stress distribution in the whole body.

Keywords: residual stress; eigenstrain; reconstruction; iterative finite element model; contour method 


\section{INTRODUCTION}

More than $65 \%$ of the energy consumed in today's world is produced using steam generated by burning fossil fuels. The highest share in fossil-fuelled power plants is coal with $60 \%$ and it is followed by gas and oil with shares of 30 and $10 \%$ respectively. The use of coal in fossil-fuelled power plants increase because of the increase in oil prices. U.S. Energy Information Agency [1] reported that although the share of renewable energy sources continuously increases, it is expected that $37 \%$ of world's total energy will be produced using coal-powered plants in 2035. Studies for efficiency improvement in coal-fired power plants focused on ultra-supercritical technology. Research in advanced ultra-supercritical technology promises efficiencies more than $50 \%$. Barnes [2] stated that this technology has a potential to achieve higher efficiencies than conventional pulverised coal combustion units by operating at increasingly higher temperatures (up to $760^{\circ} \mathrm{C}$ ) and pressures (up to $35 \mathrm{MPa}$ ). Inconel Alloy 740H, a nickel-base precipitation hardenable super-alloy, which is produced by Special Metals Corporation Company, is able to satisfy these requirements. Having high strength and creep resistance at high temperatures and providing coal ash corrosion resistance makes this material preferable for coal combustion plants.

Power generation plants operate at high temperature and pressure conditions. Fulfilling the structural integrity of their components at these conditions is an important problem. Furtado and Le May [3] stated that operating at high-temperatures emerges the deterioration processes such as fatigue strength and creep behaviour of components and their effects are enhanced by residual stresses. Accordingly, investigation of residual stress and its effect on structural deteriorations is vital for understanding the service life of super-alloys. 
Welding is a widely used technique for joining of industrial components, but it affects microstructure and chemistry of materials. Withers and Bhadeshia [4] illustrated that temperature gradients around the weld zone cause formation of residual stresses. Rapid heating results in volume expansion in weld zone while the resistance of surrounding cooler parts causes formation of thermal stresses and rapid cooling ends with the shrink of the weld bead. Finally, the process reaches a steady state of balance of tensile and compressive residual stresses in and around the weld beam.

Several non-destructive methods were developed to investigate residual stresses in welded components. Most widely used ones are X-ray and neutron diffraction techniques and they are used for investigation of surface, sub-surface and in-volume stress distribution and their applications are demonstrated in the book by Fitzpatrick and Lodini [5]. Tanala et al. [6] used sub-surface and rayleigh ultrasonic waves and Palanichamy et al. [7] used longitudinal critically refracted (LCR] ultrasonic waves for non-destructive determination of surface and sub-surface residual stresses. Javadi et al. [8] investigated the capability of LCR waves on evaluation of in-volume residual stresses using transducers that operate at different frequencies. Uzun and Bilge [9] showed that longitudinal ultrasonic waves can be used to estimate in-volume bulk residual stress throught the thickness of welded steel plates. However, diffraction techniques are expensive, provide limited amount of data and have time restrictions and besides, ultrasonic waves provide information about average residual stress in a bulk volume with low resolution. Accordingly, current techniques are not appropriate for effective mapping of three-dimensional residual stress distribution within large volumes.

The contour method was first introduced by Prime [10] for determination of planar distribution of residual stresses through the thickness of large engineering components on a 
cross-section obtained using electron discharge machining (EDM) cut. Kartal et al. [11] used this method for calculation of residual stresses in thick welded components and showed that being not affected by the microstructural variations makes it a suitable method for this type of applications. Kartal et al. [12] used neutron diffraction for residual stress investigation in welded thick specimens, Prime et al. [13] used neutron diffraction for residual stress investigation in welded thick aluminium alloys, Toparli et al. [14] used X-Ray diffraction for residual stress investigation in laser peened aluminium alloy samples, Traore et al. [15] used neutron diffraction for residual stress investigation in welded steel components and Zabeen et al. [16] used X-Ray diffraction for residual stress investigation in laser peened compressor blades for verification of residual stress evaluations performed using contour method. Carlone et. al. [17] compared experimental residual stresses obtained using the contour method with thermomechanical finite element simulation results. Previous studies showed that the contour method is a reliable technique for investigation of residual stress formed as a result of thermal processes in metals. Reliability of the contour method allows investigation of effect of residual stress on formation of further defects in the materials. Citarella et. al. [18] showed that the residual stresses obtained by the contour method allow a good match between numerical and experimental crack growth rates.

The standard contour method has two limitations. First, this method is just sensitive to the stress component normal to the cut surface. Second, displacements are only related to the residual stresses on the surface of cut. In order to overcome these limitations and to model three-dimensional residual stress distributions, DeWald and Hill [19] used displacement data obtained by contour measurements for eigenstrain reconstruction of residual stress fields within the whole body. Kartal et al. [11] also calculated in-volume residual stresses using eigenstrain reconstruction method. These attempts are based on the assumption that the 
processing of the body is uniform along one direction. Residual stress fields are calculated by reconstructing tensile and compressive eigenstrain distribution in the two-dimensional plane of cut surface and the distribution is accepted to be same along the longitudinal direction.

Welding process causes the formation of tensile residual stresses in the weld zone and compressive stresses are formed, as a result of this tensile stresses, around the weld zone. Tensile and compressive stress zones balance each other and create welding residual stress. Although eigenstrain reconstruction method was used for determination of welding residual stress in large volumes, there has been no attempt to investigate the main source of welding residual stress using eigenstrains.

In this study, weld samples are prepared using bead-on-plate specimen design. Singlecomponent and multi-component inverse eigenstrain problems are solved to determine permanent plastic strain distribution in the whole body. A new model is developed for solving the inverse eigenstrain problem to deal with difficulties that arise in the cases of lack of sufficient experimental data. Analyses done using these models allowed identification of the main source of welding residual stress in nonuniformly processed as-welded and postweld heat treated specimens.

\section{MATERIAL AND SPECIMEN DESIGN}

The materials used in this study are as-welded and heat-treated specimens of welded Inconel Alloy $740 \mathrm{H}$ which is a nickel-base, precipitation hardenable super-alloy designed for advanced ultra-supercritical power generation. It has resistance to coal ash corrosion at high temperatures as a consequence of high content of chromium, $24.5 \%$. Other elements of the 
chemical composition of this super-alloy are cobalt, $20.0 \%$, aluminium, $1.35 \%$, titanium, $1.35 \%$ and niobium, $1.5 \%$. Iron, carbon, manganese, molybdenum, silicon, copper, phosphorus, sulphur and boron elements are also included at nominal ratios and balance is satisfied by the nickel.

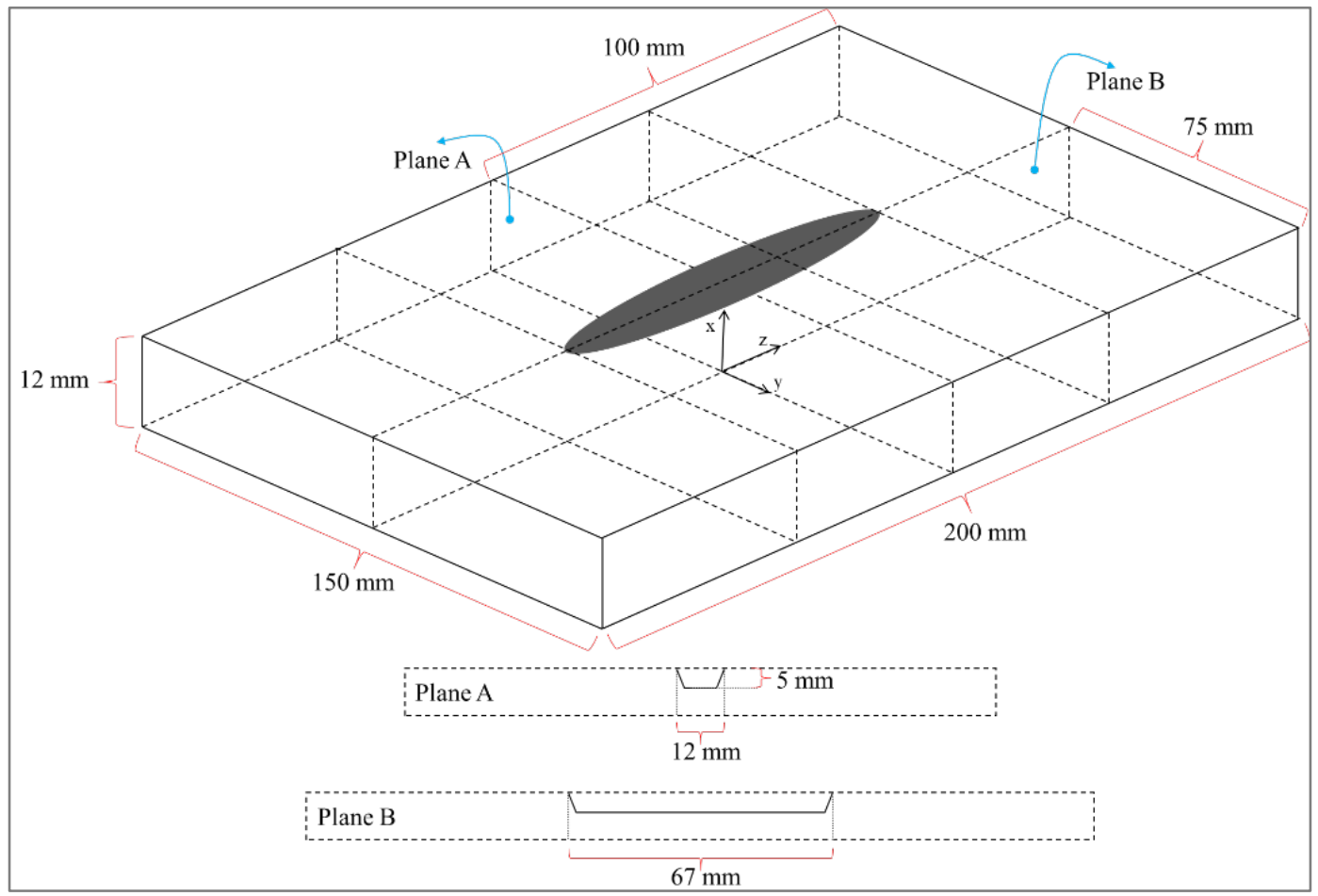

Figure 1. Three-dimensional representation of the Inconel Alloy $740 \mathrm{H}$ specimen and its dimensions.

Each specimen has a groove filled with three weld beads. Dimensions of the specimen design is illustrated in Figure 1. The thickness of plates is $12 \mathrm{~mm}$ while their length and width are 200 and $150 \mathrm{~mm}$ respectively. The slot for weld has approximately $12 \mathrm{~mm}$ width and $67 \mathrm{~mm}$ length at the top surface and $8 \mathrm{~mm}$ width and $63 \mathrm{~mm}$ length at its bottom section. After the weld slots are machined, samples are pre-weld annealed at $1107^{\circ} \mathrm{C}$ for 1 hour with water cooling to reduce the residual stresses introduced by the machining process. 
Chemical composition of the filler metal is the same as base plates. Three weld beads are applied with the same sequence using tungsten inert gas (TIG) welding technique. Parameters of the welding process are given in Table 1. One of the welded samples is left as-welded and the other sample is post weld heat treated (aged) at $800{ }^{\circ} \mathrm{C}$ for 4 hours with air cooling. The $\mathrm{x}-\mathrm{y}-\mathrm{z}$ coordinate system origin is located in the plane of the bottom surface of each specimen at the geometric centre of the specimen as illustrated in Figure 1. The welding direction is determined to be the positive $\mathrm{z}$-axis. Weld arc approximately starts to travel from $\mathrm{z}=-33.5$ $\mathrm{mm}$ and stops at $\mathrm{z}=33.5 \mathrm{~mm}$ along weld centre line on the top surface.

Table 1. Parameters for TIG welding of as-welded (AW) and heat treated (HT) specimens.

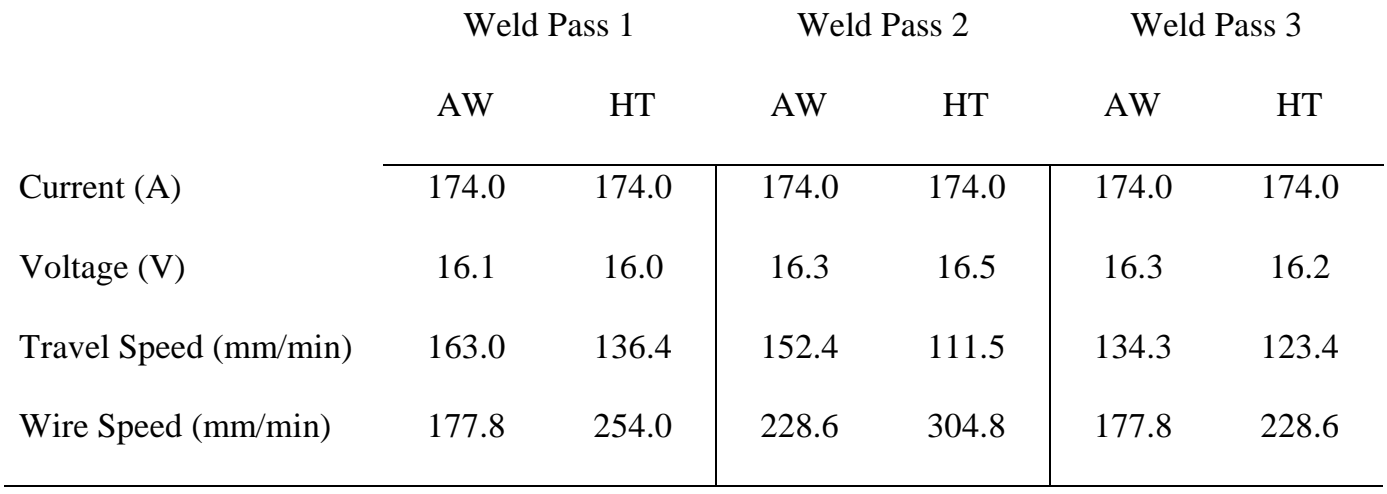

\section{THE CONTOUR METHOD}

Residual stresses cannot be measured directly but can be quantified using experimental measurements and suitable models. The contour method provides experimental data that can be interpreted by a finite element model to correlate them with stress. This destructive method is based on creating a contour map of a cut surface, which is distorted as a consequence of residual stresses, using profilometry. Setting the contour back to the flat condition brings residual stresses, that were released during cutting, back. This process can be 
simulated by imposing the inverse of the measured and processed displacements using a finite element model.

Previous applications of the contour method are based on the assumption that stress distribution in the direction perpendicular to the surface of cut is assumed to be uniform because of the lack of necessary data to reconstruct variations of eigenstrain in the whole body. This limitation prevented application of this method to complex structures. Another limitation of this method is related to the cutting technique. Electric discharge machining (EDM) sectioning process does not allow determination of displacements parallel to the cut plane by coordinate measuring machine (CMM). Accordingly, the only single-component of residual stress which is normal to the cut surface can be calculated based on the principle of superposition which assumes that stresses normal to the cut-surface are relaxed elastically after the cutting process. This linear relationship between displacement and stress turns residual stress calculations of the contour method into a reliable process.

In this study, as-welded and heat-treated specimens of Inconel alloy $740 \mathrm{H}$ are cut from Plane A using AgieCharmilles CUT 20 P Wire EDM cutting machine at cutting speeds of 2.0 and $2.2 \mathrm{~mm} / \mathrm{min}$ respectively. Diameter of the wire used for this process is determined to be 0.25 mm. Contour measurements on both surfaces of the cuts are performed using Crysta Apex C 3D CNC CMM with a density of 200 measurement points per $\mathrm{mm}^{2}$. In addition to surface measurements, perimeters of all four parts around the cut surface are determined by coordinate measurements and recorded for data analysis. The machine used for this process has accuracy of $1.7 \mu \mathrm{m}$ and resolution of $0.1 \mu \mathrm{m}$. The collected data are first analysed and then used for finite element stress calculations. The data analysis process is consisted of aligning, interpolating to a common grid, extrapolating to the perimeter, averaging, cleaning, 
smoothing and flattening stages. For the sake of simplicity, details of the well-known data analysis steps are not given in the text.

\section{THE INVERSE EIGENSTRAIN MODEL}

Residual stress are the remaining stresses after the end of a loading state. Korsunsky [20] stated that deformation behaviour and fatigue durability of engineering components and assemblies cannot be understood without proper information about residual stresses within a structure. They usually have a complex nature of arbitrarily shaped bodies and difficulty arises in the determination of the six components of the residual stress tensor. Ahn et al. [21] explain that it is difficult to create an experimental procedure to obtain this level of detail while current experimental methods for determination of welding residual stresses are expensive and require a long time of labour force. In order to deal with this problem, computational welding simulations are used that can predict distribution and magnitude of residual stresses. However, current methods require sensitive calibration of measurable parameters, which are weld geometry and temperature distribution, and highly accurate temperature dependent thermal-structural material properties to conduct elastic-plastic finite element simulations.

Without any outer loading, residual stresses are self-equilibrating. Elastic deformations cannot form residual stresses, but permanent inelastic strains, which are formed after manufacturing processes, cause the formation of residual stress fields. Mura [22] called the permanent plastic strain in materials as eigenstrain. If the sole source of residual stresses, eigenstrains, are introduced into a body, they do not fit properly, but the fit is accommodated by elastic strains between them. This linear problem can be solved to calculate residual stress 
fields within a body without the requirement of complex material properties and calibration procedures of elastic-plastic models. Examples of this process can be found in the book of Korsunsky [20]. Solving the direct eigenstrain problem allows calculation of residual stresses using a given eigenstrain field while solving the inverse eigenstrain problem provides the eigenstrain field that is the reason of a known residual stress distribution or a measurable value like displacements caused by residual stress relaxation.

Sectioning or cutting a residual stress including body causes relaxation of stresses, but eigenstrains are not affected. This property of eigenstrains allows modelling of cutting process of a material. If displacements related to stress relaxation after the cutting process are known, they can be used to formulate the inverse eigenstrain problem. In this study, residual stresses in a nonuniform body are calculated by determining the eigenstrain distribution that provides displacements fitting with experimentally measured displacements coming from relaxation after cutting process. The use of precious displacement data measured from the surface of cut turns eigenstrain reconstruction process into a model that validates itself with experimental results.

The eigenstrain reconstruction of residual stress distribution requires accurate determination of boundaries of eigenstrain fields. To achieve this target, the basis-functions are created by multiplying Chebyshev polynomial functions. The maximum order of polynomials is determined to be 5 in all proposed models. In order to obtain eigenstrain fields formed solely in and around the weld zone, distribution of the basis-functions is limited by Gauss function. Shape of the Gauss function is analysed to satisfy the desired fit. The distribution of Chebyshev polynomials up to $5^{\text {th }}$ order between -1 and 1 interval after combining with the Gauss function are given in Figure 2. 


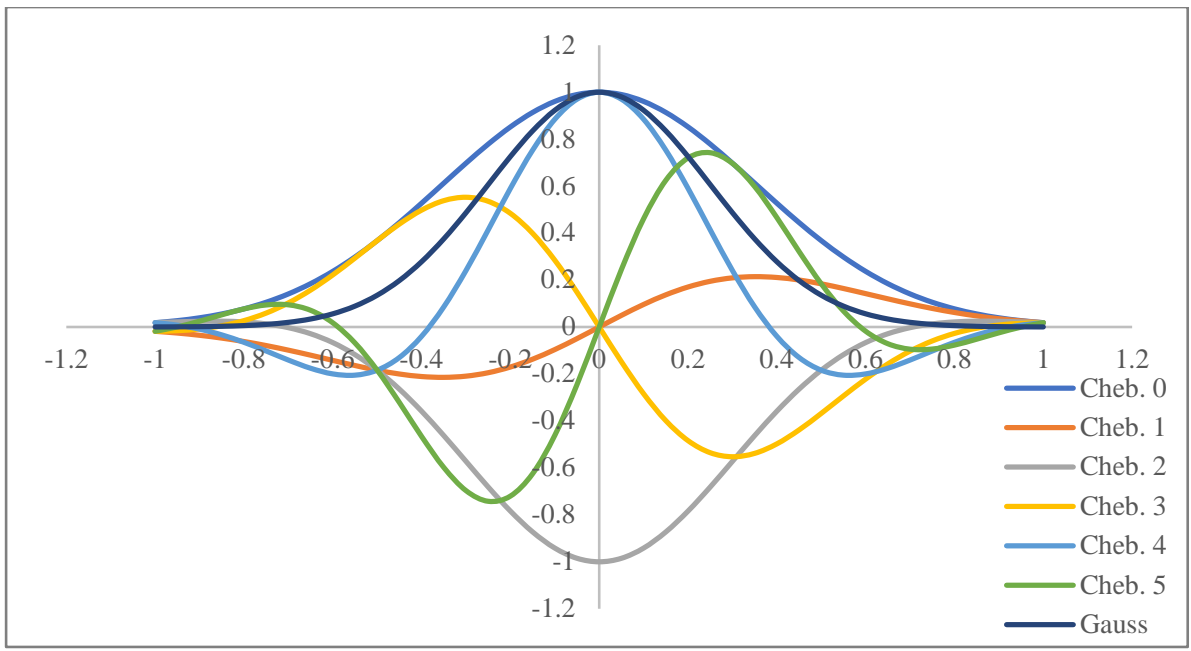

Figure 2. Chebyshev functions up to $5^{\text {th }}$ order combined with Gauss functions.

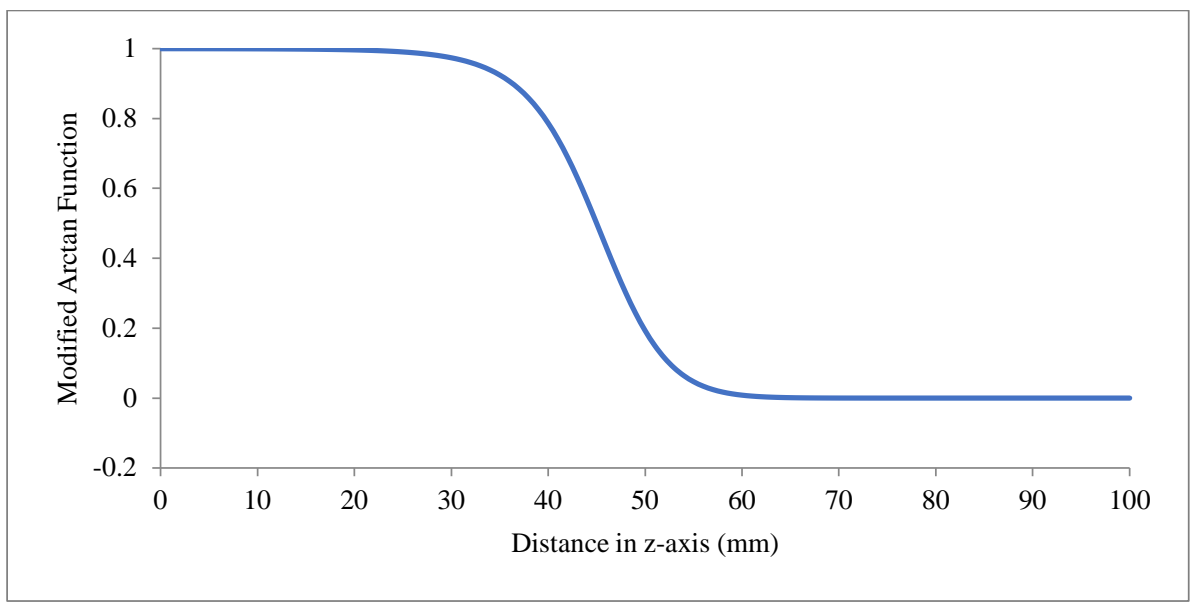

Figure 3. Modified Arctan function that defines the length of weld beam.

Bead on plate weld design provides beneficial advantages on the understanding of different stages of the welding process, but modelling of this design has some difficulties. The process of eigenstrain reconstruction of residual stresses uses the assumption that eigenstrain is uniform in one principal direction that is the longitudinal direction in the bead on plate design; however, weld beam in this design is not uniform. In order to model weld beam length while keeping the uniform distribution of eigenstrain, modified Arctan function is applied in the longitudinal direction as illustrated in Figure 3. 
The degree of fit obtained after the solution of inverse eigenstrain problems are quantified using mean squared error (MSE) estimation. This statistical method is used to compare experimental displacements and residual stresses calculated using contour method with displacements and residual stresses calculated using inverse eigenstrain reconstruction methods. The quantified magnitude of fit is accepted to be satisfactory if it is closer to zero. MSE of each solution is determined using the equation given below:

$M S E=\frac{1}{n} \sum_{p=1}^{r}\left(Y_{p}^{2}-X_{p}^{2}\right)$

where $Y_{p}$ is the calculated displacement or residual stress using inverse eigenstrain reconstruction method and $X_{p}$ is the measured and averaged displacement or calculated residual stress using contour method in the $p^{\text {th }}$ measurement point.

Three different models for the eigenstrain problem are used for modelling of a nonuniform body. The single-component model finds the distribution of the single-component of eigenstrain that is parallel to the residual stresses relaxed after the cutting process. Multicomponent models determine the distribution of two components ( $\mathrm{zz}$ and yy components) of eigenstrain which are parallel and normal to the weld beam. All models reconstruct eigenstrain distribution in the whole body using Chebyshev polynomial, Gauss and modified arctan functions. Chebyshev polynomials create a planar distribution of eigenstrains. Multiplication of this planar distribution by Gauss function allows determination of the distribution limits of eigenstrains. Distribution size determined using Gauss function is analysed in terms of quantified quality of fit of model results with experimental data. The shape of modified arctan function, used for adjusting the length of weld zone, is determined 
by a trial and error process in a way to obtain the best fit and kept constant at all simulations. As illustrated in Figure 3, this function varies parallel to the weld direction and modifies eigenstrains in each plane according to their depth along the longitudinal direction.

\subsection{Single-Component Model}

In the case of determination of single-component of unknown eigenstrain distribution, uniform $z z$ component of eigenstrain is defined along the longitudinal direction.

$\varepsilon^{*}=\left[\begin{array}{ccc}0 & 0 & 0 \\ 0 & 0 & 0 \\ 0 & 0 & \varepsilon_{z z}^{*}\end{array}\right]$

According to the superposition principle, deformations in an elastic body are directly proportional to the deforming force and, accordingly, a series expansion can be used to define the two-dimensional distribution of eigenstrain:

$\varepsilon^{*}(x, y)=\sum_{l=1}^{(m+1)(n+1)} A_{l}^{z z} F_{l}(x, y)$

where $A_{l}^{Z Z}$ is the unknown coefficient of $l^{\text {th }}$ term of a series expansion of known basis funcions, $F_{l}(x, y)$. The basis functions are used to generate planar distribution of eigenstrain by multiplying Chebyshev polynomial functions:

$F_{l}(x, y)=P_{l, i}(x) P_{l, j}(y)$ 
In this equation, $i$ ranges from 0 to $m$ and $j$ ranges from 0 to $n$ while $m$ and $n$ define the maximum order of polynomial functions. Total of unknown coefficients of the basis functions, $w$, is determined as given below:

$w=(m+1)(n+1)$

The inverse eigenstrain problem, which is an elastic problem, solved to calculate the unknown coefficients using the displacement data from contour measurements:

$\{u\}=\left[C^{S}\right]\left\{A^{S}\right\}$

where $\{u\}$ is a vector containing measured average displacements on the contour cut surface at $r$ number of measurement points:

$\{u\}=\left[u_{1}(x, y), u_{2}(x, y), \ldots, u_{r}(x, y)\right]^{T}$.

The vector $\left\{A^{S}\right\}$ contains coefficients of $w$ number of basis functions:

$\left\{A^{S}\right\}=\left[A_{1}^{Z Z}, A_{2}^{z Z}, \ldots, A_{w}^{Z Z}\right]^{T}$.

The model derived the matrix of single-component solution, $\left[C^{S}\right]$, which is composed of displacements obtained by solving basis functions. The matrix has $w$ number of columns for each polynomial term and $r$ number of rows corresponding to each individual data point. The terms of basis functions are used to define eigenstrain distribution in finite element 
simulation whereas the resulting deformations at equilibrium were stored to create the influence matrix, $\left[C^{S}\right]$.

$$
\left[C^{S}\right]=\left[\begin{array}{ccc}
C_{1,1}^{Z Z} & \cdots & C_{w, 1}^{z Z} \\
\vdots & \ddots & \vdots \\
C_{1, r}^{z Z} & \cdots & C_{w, r}^{z Z}
\end{array}\right]
$$

The unknown vector of coefficients, $\left\{A^{S}\right\}$, is calculated using least squares fit process that minimizes the error between the model-derived and the measured displacement data:

$$
\left\{A^{S}\right\}=\left(\left[C^{S}\right]^{T}\left[C^{S}\right]\right)^{-1}\left[C^{S}\right]^{T}\{u\}
$$

\subsection{Multi-Component Model}

According to the principle of superposition, in the case of multi-component inverse eigenstrain problem, eigenstrains that cause deformations in an elastic body are the sum of separate components of eigenstrain:

$$
\varepsilon^{*}=\left[\begin{array}{ccc}
0 & 0 & 0 \\
0 & \varepsilon_{y y}^{*} & 0 \\
0 & 0 & 0
\end{array}\right]+\left[\begin{array}{ccc}
0 & 0 & 0 \\
0 & 0 & 0 \\
0 & 0 & \varepsilon_{z z}^{*}
\end{array}\right]
$$

where $\varepsilon^{*}$ is the eigenstrain, $\varepsilon_{y y}^{*}$ is the yy component of eigenstrain and $\varepsilon_{z z}^{*}$ is the $z z$ component of eigenstrain. The principle of superposition also enables determination of the separate components of eigenstrain as sum of linear combinations of unknown polynomial coefficients with independent coefficients: 
$\varepsilon^{*}(x, y)=\sum_{k=1}^{(m+1)(n+1)} A_{k}^{y y} F_{k}(x, y)+\sum_{l=1}^{(m+1)(n+1)} A_{l}^{z z} F_{l}(x, y)$

where $A_{k}^{y y}$ and $A_{l}^{z z}$ are the unknown coefficients of $k^{t h}$ and $l^{\text {th }}$ terms of a series expansion of known basis functions:

$F_{k}(x, y)=P_{k, i}(x) P_{k, j}(y)$

$F_{l}(x, y)=P_{l, i}(x) P_{l, j}(y)$

Similar to the single-component inverse eigenstrain problem, the unknown coefficients are calculated using Equation 15:

$\{u\}=\left[C^{M}\right]\left\{A^{M}\right\}$

The vector $\left\{A^{M}\right\}$ contains coefficients of $2 w$ number of basis functions of multi-component inverse eigenstrain problem:

$\left\{A^{M}\right\}=\left[A_{1}^{y y}, A_{2}^{y y}, \ldots, A_{w}^{y y}, A_{1}^{z z}, A_{2}^{z z}, \ldots, A_{w}^{z z}\right]^{T}$

The model derived matrix, $\left[C^{M}\right]$, is composed of displacements obtained by solving basis functions, $F_{k}(x, y)$ and $F_{l}(x, y)$. The matrix used for multi-component inverse eigenstrain problem has $2 w$ number of columns for each polynomial term and $r$ number of rows corresponding to data points. 
$\left[C^{M}\right]=\left[\begin{array}{cccccc}C_{1,1}^{y y} & \cdots & C_{w, 1}^{y y} & C_{1,1}^{z z} & \cdots & C_{w, 1}^{z z} \\ \vdots & \ddots & \vdots & \vdots & \ddots & \vdots \\ C_{1, r}^{y y} & \cdots & C_{w, r}^{y y} & C_{1, r}^{z z} & \cdots & C_{w, r}^{z z}\end{array}\right]$

The vector of unknown coefficients, $\left\{A^{M}\right\}$, is determined using Equation 18. After determination of the coefficients, the eigenstrain distribution is calculated using Equation 12 and they are input into a finite element model as pseudo-thermal eigenstrain to get an estimation of the initial residual stress distribution before cutting process. Eigenstrain components other than $\varepsilon_{y y}^{*}$ and $\varepsilon_{z z}^{*}$ are assumed to be zero.

$\left\{A^{M}\right\}=\left(\left[C^{M}\right]^{T}\left[C^{M}\right]\right)^{-1}\left[C^{M}\right]^{T}\{u\}$

\subsection{Multi-Component Iterative Model}

The primary component of eigenstrain that drives the model for reconstruction of residual stresses is the zz-component. In order to get better fit of experimental measurements with the model calculations, authors concluded that yy-component of eigenstrain should be included. For this purpose, the multi-component solution is ideal from the point of view of flexibility, but it requires additional experimental data, that provides information about residual stresses that distribute along the longitudinal direction of the weld, to determine $z z$ and yy components of eigenstrain independently. In order to include the second component of eigenstrain while keeping the model stable and computationally efficient, the problem is regularised by making a physically-based assumption that $z z$ and yy components of eigenstrain are proportional with a fixed coefficient and an iterative solution process of the multi-component eigenstrain problem is developed. In this process, separate components of eigenstrain are multiplied by independent coefficients which are $\alpha$ for $\varepsilon_{y y}^{*}$ and $\beta$ for $\varepsilon_{z z}^{*}$ : 
$\varepsilon^{*}=\alpha\left[\begin{array}{ccc}0 & 0 & 0 \\ 0 & \varepsilon_{y y}^{*} & 0 \\ 0 & 0 & 0\end{array}\right]+\beta\left[\begin{array}{ccc}0 & 0 & 0 \\ 0 & 0 & 0 \\ 0 & 0 & \varepsilon_{z z}^{*}\end{array}\right]$

Separate components of eigenstrain are determined as the sum of linear combinations of unknown polynomial series with same coefficients.

$\varepsilon^{*}(x, y)=\alpha\left(\sum_{f=1}^{(m+1)(n+1)} A_{f}^{I} F_{f}(x, y)\right)+\beta\left(\sum_{f=1}^{(m+1)(n+1)} A_{f}^{I} F_{f}(x, y)\right)$

where $A_{f}^{I}$ is the unknown coefficient of $f^{\text {th }}$ term of a series expansion of known basis functions:

$F_{f}(x, y)=P_{f, i}(x) P_{f, j}(y)$

The elastic problem is solved again using the measured average displacements to calculate the unknown coefficients:

$\{u\}=\left[C^{I}\right]\left\{A^{I}\right\}$

The vector $\left\{A^{I}\right\}$ contains coefficients of $w$ number of basis functions of multi-component iterative procedure for inverse eigenstrain problem:

$\left\{A^{I}\right\}=\left[A_{1}^{I}, A_{2}^{I}, \ldots, A_{w}^{I}\right]^{T}$ 
The model derived matrix, $\left[C^{I}\right]$, is composed of displacements obtained by solving basis functions, $F_{f}(x, y)$. The matrix used for multi-component iterative process has $w$ number of columns for each polynomial term and $r$ number of rows corresponding to each individual data point.

$\left[C^{I}\right]=\left[\begin{array}{ccc}C_{1,1}^{I} & \cdots & C_{w, 1}^{I} \\ \vdots & \ddots & \vdots \\ C_{1, r}^{I} & \cdots & C_{w, r}^{I}\end{array}\right]$

In the next stage of iterative process, coefficients of independent solutions of yy and $z z$ component of eigenstrain, $\left\{A^{I I}\right\}$, are calculated using common coefficients of basis functions, $\left\{A^{I}\right\}$, by solving the elastic inverse eigenstrain problem:

$\{u\}=\left[C^{I I}\right]\left\{A^{I I}\right\}$

The vector $\left\{A^{I I}\right\}$ contains coefficients $\alpha$ and $\beta$ for independent solutions.

$\left\{A^{I I}\right\}=[\alpha, \beta]^{T}$

The model derived matrix, $\left[C^{I I}\right]$, is composed of displacements obtained by solving $\varepsilon^{*}(x, y)$ for $\varepsilon_{y y}^{*}$ and $\varepsilon_{z z}^{*}$ independently which are done by setting $\alpha$ and $\beta$ to zero while solving for $\varepsilon_{z z}^{*}$ and $\varepsilon_{y y}^{*}$ respectively.

$\left[C^{I I}\right]=\left[C_{\alpha}, C_{\beta}\right]$ 
Flowchart of the iterative solution process for the multi-component problem is given in Figure 4. The main solution includes calculation of the coefficients of basis-functions and the coefficients defined for each component of eigenstrain. At the end of a solution, displacements are compared with previous solution and a decision is made on the continuation of the iteration process. If the difference between the final and previous solutions at an acceptable rate, iteration process is terminated. Otherwise, the coefficients of each component of eigenstrain are recorded and a new solution is started.

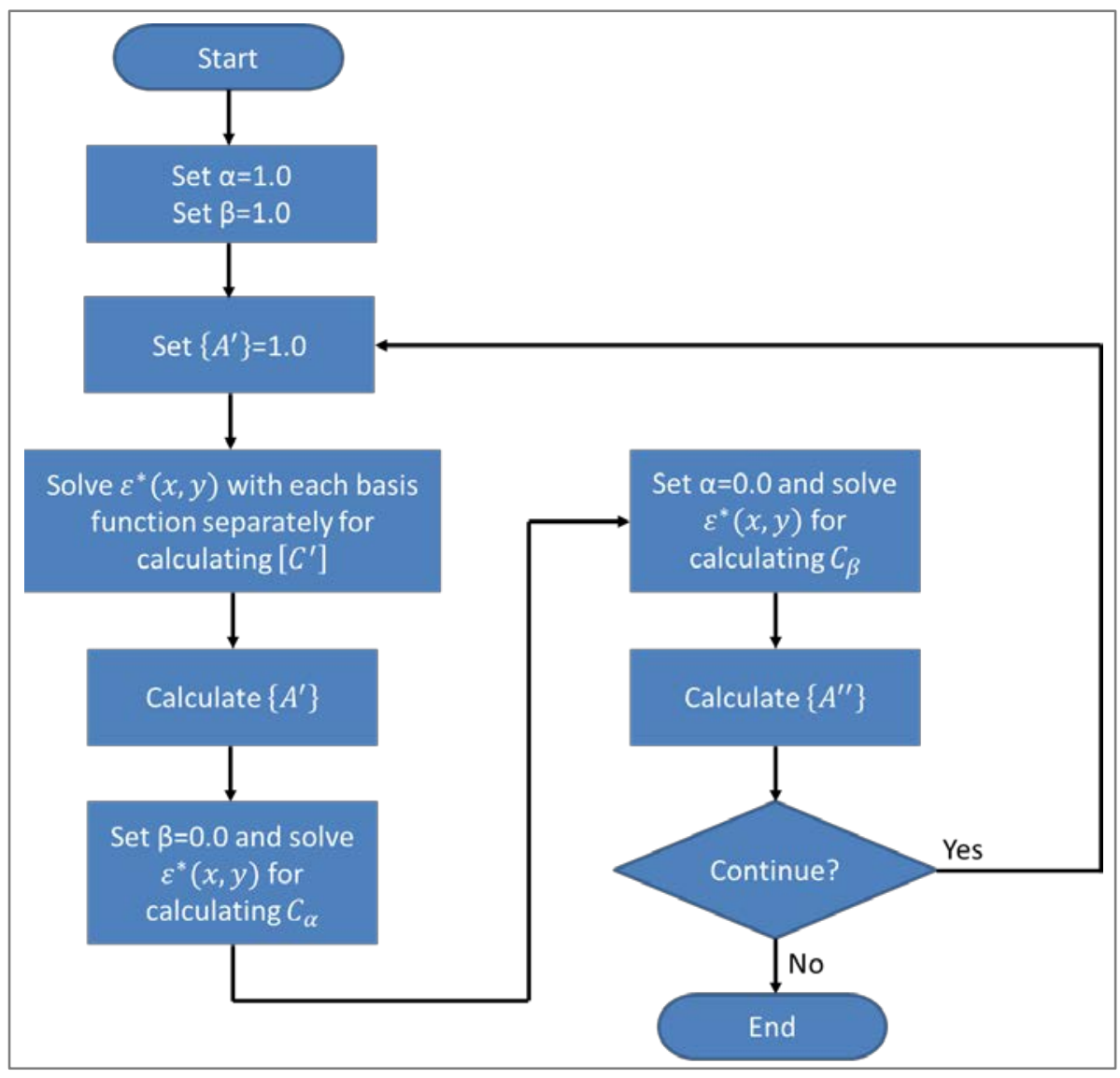

Figure 4. Flowchart of the iterative solution process. 


\section{RESULTS}

Distribution of eigenstrain in as-welded and heat-treated specimens of Inconel 740H superalloys are investigated by solving the inverse eigenstrain problem using three different models. Simulations are performed at different eigenstrain distribution scales while keeping parameters the same for all solutions. Results are analysed in terms of the quantified quality of fit based on MSE calculations. The model is verified using profile plots of experimentally measured displacements and elastic stress calculations of the contour method.

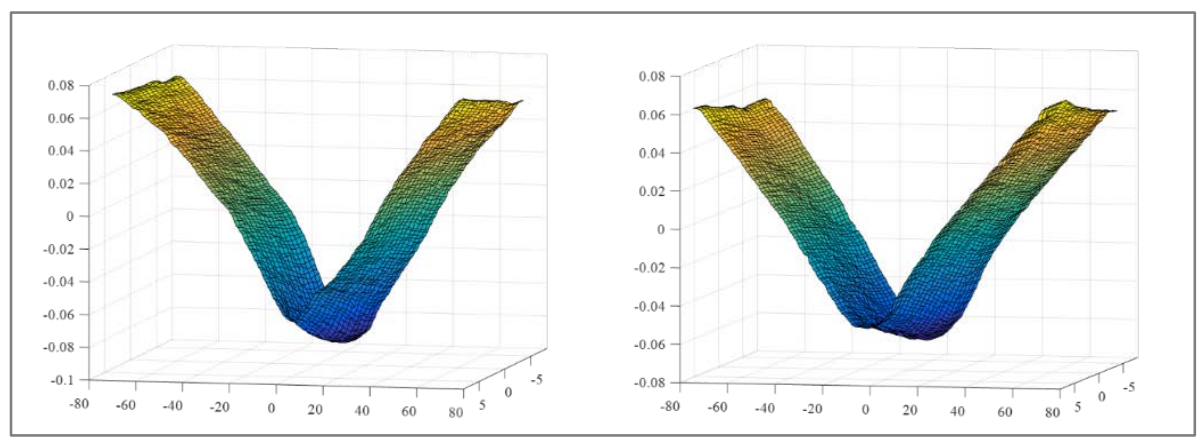

Figure 5. The processed displacement data of as-welded (left) and heat-treated (right) specimens used for finite element models of contour method and inverse eigenstrain problem.

The displacement data used for reconstruction process and experimental longitudinal residual stresses are obtained using contour method. This method is composed of two main stages which are data processing of experimentally measured data and finite element modelling. In this study, data processing is performed using computational tools of MATLAB and modelling is done using ABAQUS commercial finite element analysis software. Data processing stage creates the displacement data sets, which are used for contour method residual stress calculations and inverse eigenstrain problem, by processing raw data of displacement measurements of the CMM. The processed displacement data used in as-welded 
and heat-treated contour and inverse eigenstrain models are illustrated in Figure 5. As it is expected, heat-treated specimen has less deformation after post weld heat treatment process as a result of stress relaxation in the weld zone.

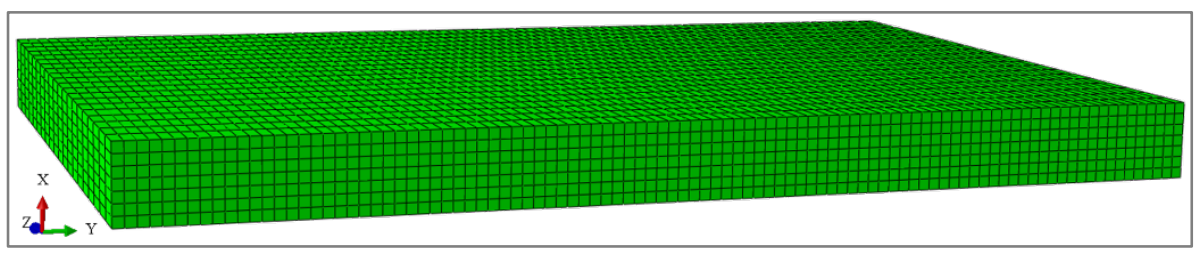

Figure 6. Illustration of model geometry and mesh distribution used for both contour method and inverse eigenstrain problem.

Solution time is an important factor that affect cost and quality of the research. There are two main factors that affect solution time of the models used in this study which are maximum order of polynomial functions and mesh density. Another factor is the number of integration points of elements. Its effect has been minimized by using C3D8R general purpose brick element with reduced integration point. Another factor that has a minor effect on the solution time is shape of elements. In order to get perfectly distributed cubic elements, a cuboid shape of the samples is created and the same volume is used for both as-welded and heat-treated models. The use of perfectly distributed elements provided smooth and efficient solutions without creating convergence problems related to mesh distribution. Observations showed that 13720 perfectly aligned cubic elements provided good fit of displacement and residual stress while the maximum order of polynomials is 5 . The same FEM model geometry and meshes are used for both contour and inverse eigenstrain methods which is illustrated in Figure 6 where xy plane on the front face represents cut surface of the specimens. 
Displacements corresponding to each nodal connection is determined on the surface of cut of contour model using cubic spline function which is created using MATLAB during the data analysis process for the purpose of smoothing. Solution of inverse eigenstrain problem requires separate solutions for all function combination. $5^{\text {th }}$ order solution requires 37 separate solutions. Solutions are performed using Intel i7 CPU with 4 cores using parallelization option of ABAQUS. Each separate solution requires 5 minutes of solution time when this mesh distribution is used and 4 cores of the CPU are parallelized. Single or multi component solutions does not make a significant change on solution time of each separate solution. Total solution time of all three models are observed to be around 3 hours.

\subsection{The Single-Component Solution}

Single-component model is based on the determination of eigenstrain fields using a singlecomponent of eigenstrain which is normal to the cut surface. This formulation of the problem is used to reconstruct surface and in-volume residual stresses in as-welded and heat-treated specimens. For the purpose of investigation of the main source of the welding residual stress, solutions at different eigenstrain distribution sizes are analysed. The distribution size of eigenstrain field in each solution are determined using Gauss function while keeping centre of the Gauss function same as the plane of cut. Distribution sizes less than $50 \mathrm{~mm}$ created a non-convergence problem and eigenstrains reached to the boundaries of the model geometry when its length is $150 \mathrm{~mm}$. Accordingly, distribution sizes from 50 to $150 \mathrm{~mm}$ with a step size of $10 \mathrm{~mm}$ are tested in separate solutions using both as-welded and heat-treated models. All solutions determine the eigenstrain distribution that provide the best possible fit of displacement with experimentally measured displacements. The quality of fit of displacement and residual stress with experimental measurements are quantified by MSE calculations. 
Variation of quantified quality of fit in terms of MSE is illustrated for displacement and residual stress in Figure 7. Each point represents the error between the model calculations and the experimental measurements. Results of the as-welded model shows that the error decreases and the quality of fit increases with rising distribution size up to $90 \mathrm{~mm}$, which is the best distribution size, and after that point, a slight increase occurs with each rise in distribution size. Similar behaviour is observed in the heat-treated model but in this case, the decrease occurs after the best distribution obtained at $110 \mathrm{~mm}$ and the magnitude of the decrease is very low when compared to the as-welded model. The reason for this behaviour is the magnitude of eigenstrains in the heat-treated model are lower and their distribution is wider than the as-welded model as illustrated in Figure 10.

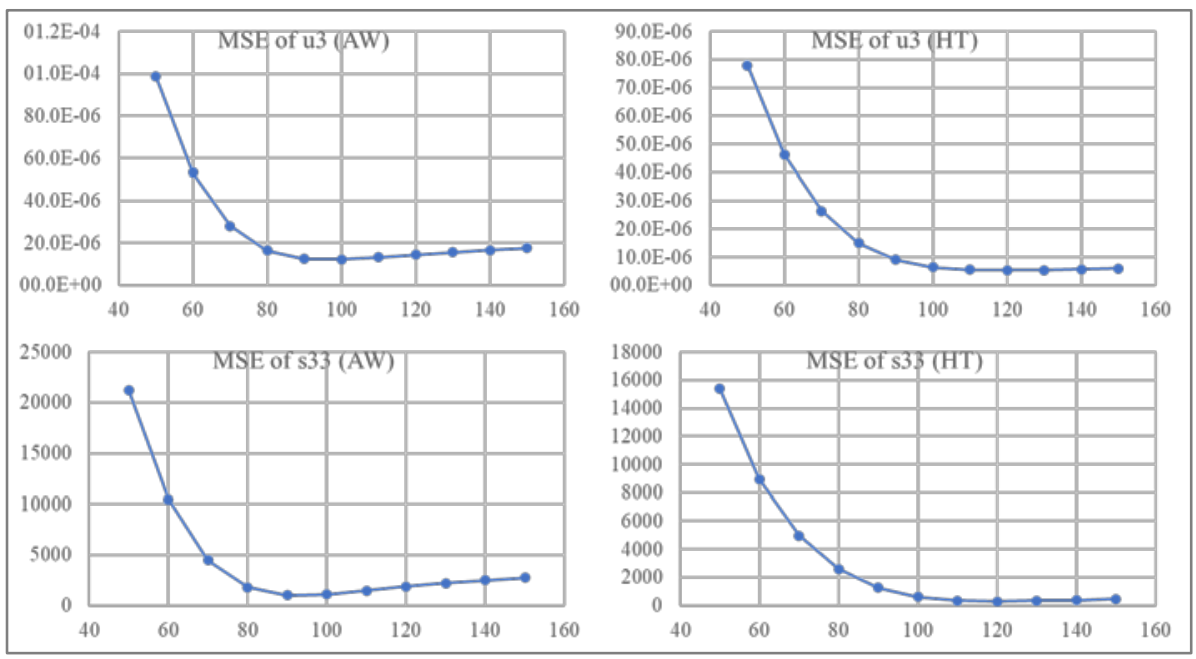

Figure 7. Mean squared error (MSE) of longitudinal displacement (u3) and residual stress (s33) w.r.t eigenstrain distribution width (in $\mathrm{mm}$ ) on the cut surface of as-welded (AW) and heat-treated (HT) samples after single-component eigenstrain reconstruction process. 


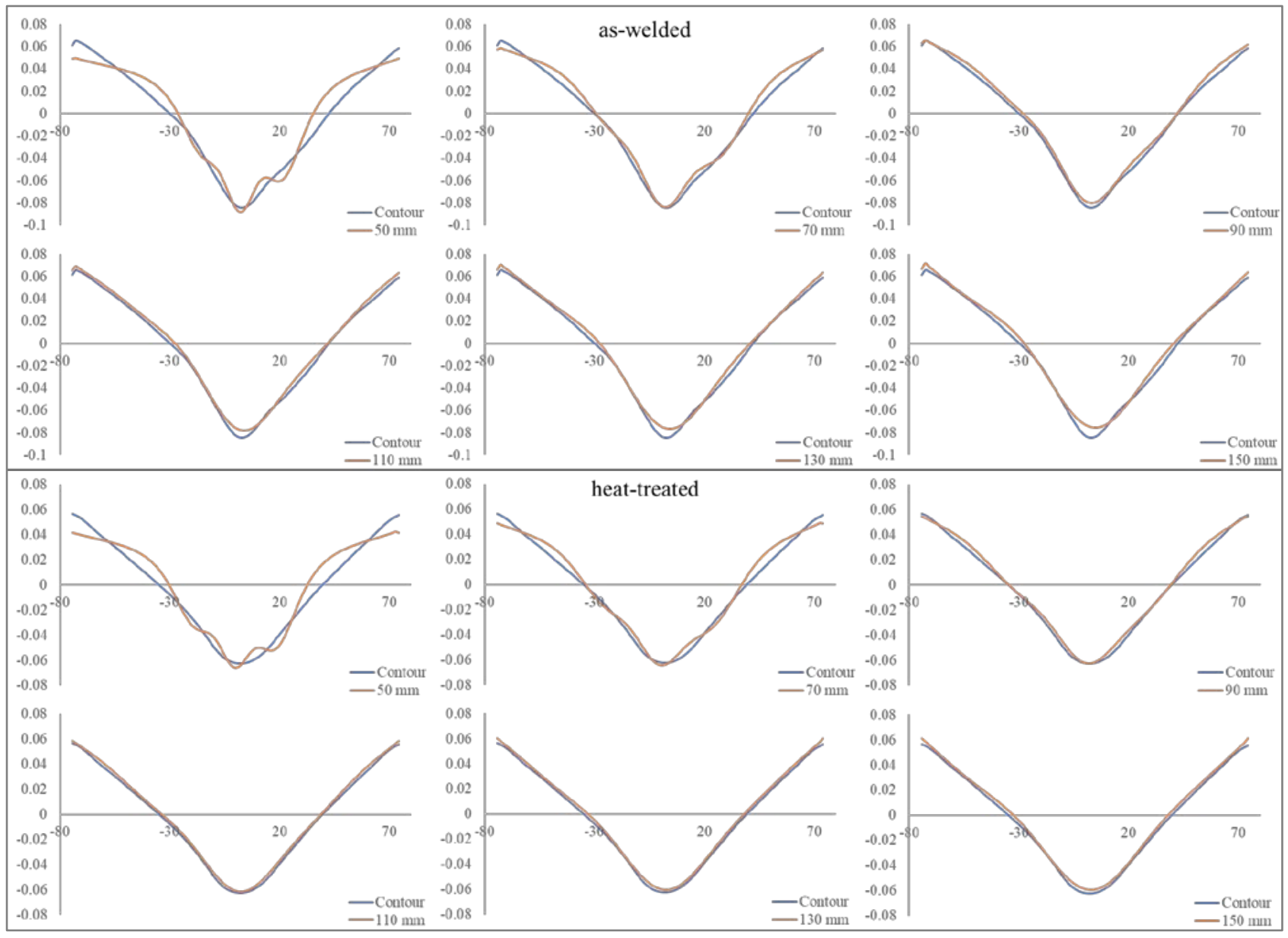

Figure 8. Transversal profile distributions of the through-thickness average of displacement (in $\mathrm{mm}$ ) obtained using single-component eigenstrain reconstruction process at different eigenstrain distribution widths from 50 to $150 \mathrm{~mm}$ and experimental results of contour method on the cut surface of the as-welded (top) and heat-treated (bottom) specimens.

Profile plots of through the thickness averaged results of single-component model and experimental results of contour method are illustrated in Figures 8 and 9. In order to show the decision-making process, 6 different solution at different distribution sizes that include the best solution are given. These visual representations are consistent with the quantified quality of fit. At shorter distribution sizes, displacement fit is very coarse. Improvement on the fit with increasing distribution size is clearly visible. Distributions higher than the best sizes have corruption of fit mostly in the central part of the model where weld zone is located. 
Similar behaviour is observed with residual stress profile plots. When the distribution is reached to the optimum size, tension stresses cause the formation of compressive stresses at the edge parts. On the other hand, distributions less than the optimum size are not able to form compressive stresses at the edge parts and, accordingly, a wing-like profile distribution appears. With increasing distribution, wing behaviour gets weaker and disappears when the optimum distribution size is used. Corruption of fit appears in the central part and extends with increasing distribution size after the optimum and this is more visible in heat treated model because of the magnitude and distribution of eigenstrain. When the magnitude of eigenstrains are higher and distribution size is lower, corruption of fit is higher, because of formation of sharper eigenstrain fields within the distribution zone.

Eigenstrain distribution in as-welded and heat-treated single-component models are illustrated in Figure 10. Dark red parts illustrate the parts with no eigenstrain. As it is expected, compressive eigenstrains have a high magnitude in the weld zone and they have consistent distribution with residual stresses. Distribution size of the heat-treated model is wider than as-welded model while the magnitude of eigenstrain in weld zone is higher in the as-welded model. Results show that heat treatment process successfully reduces the magnitude of eigenstrains but extends the distribution size of permanent plastic strains. Dong et. al. [23] and Wang et. al. [24] showed that the main mechanism behind relaxation of residual stresses is the conversion of elastic strains into creep strains. Accordingly, reason for this extension can be stated as formation of creep strains during the post weld heat treatment process. 


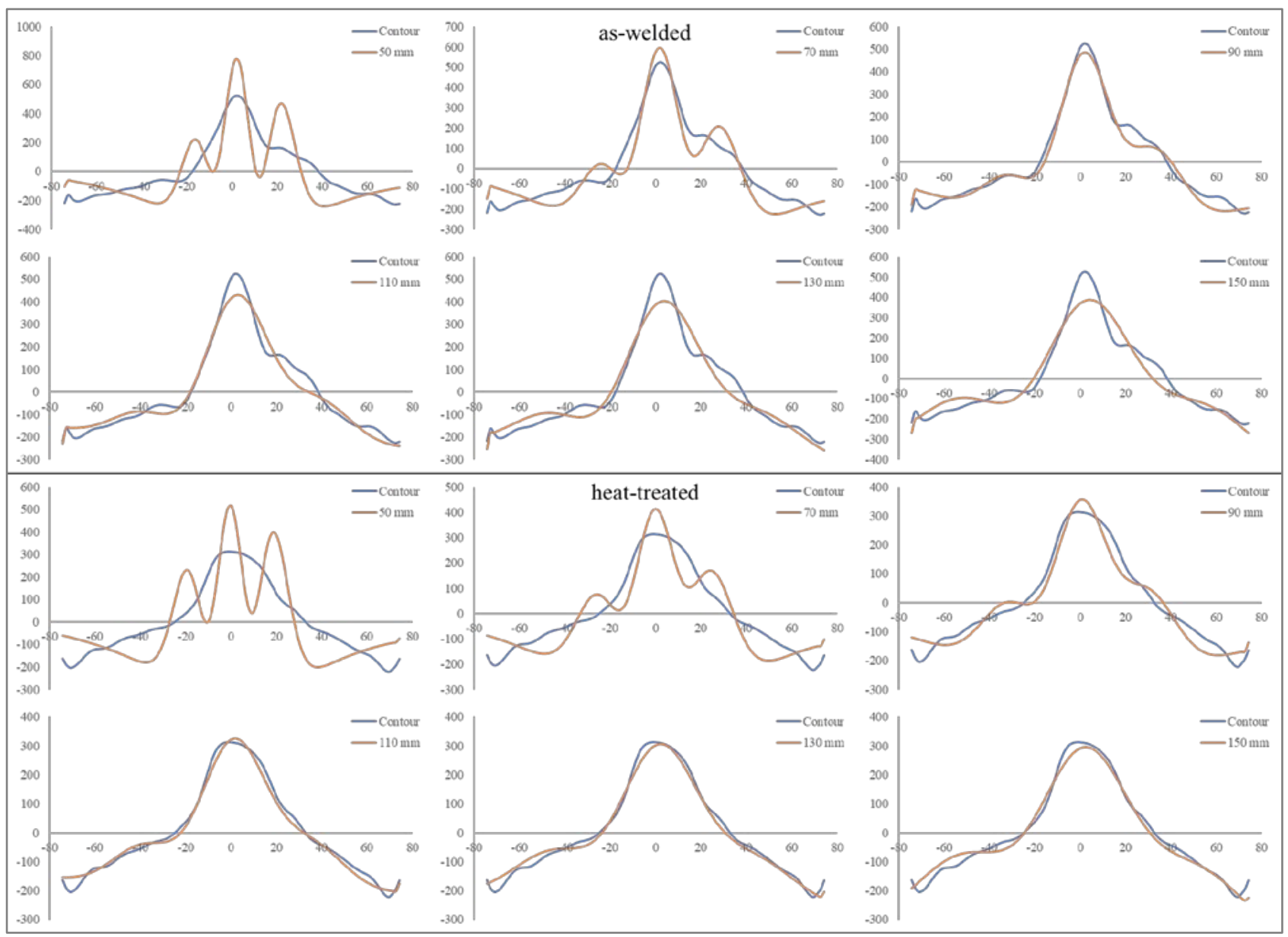

Figure 9. Transversal profile distributions of the through-thickness average of longitudinal residual stress (in $\mathrm{MPa}$ ) obtained using single-component eigenstrain reconstruction process at different eigenstrain distribution widths from 50 to $150 \mathrm{~mm}$ and experimental results of contour method on the cut surface of the as-welded (top) and heat-treated (bottom) specimens. 


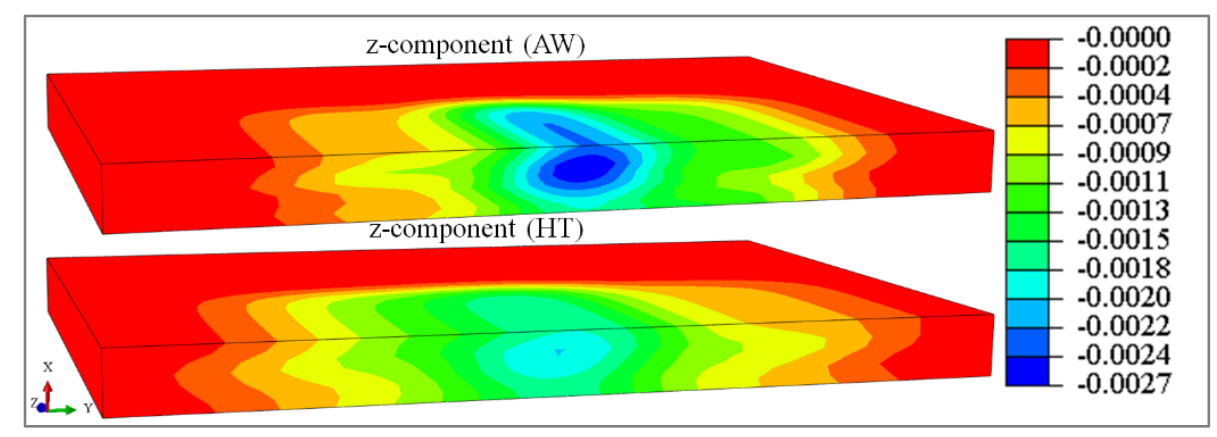

Figure 10. 3D distribution of $\varepsilon_{z z}^{*}$ in as-welded and heat-treated models, determined using single-component eigenstrain reconstruction process.

\subsection{The Multi-Component Solution}

The classical approach for eigenstrain reconstruction of residual stresses in a body is determining eigenstrain distribution using components relative to the experimental data. In spite of the fact that this approach provides an eigenstrain distribution that creates residual stresses which are good agreement with expected residual stress distribution, the second component of eigenstrain normal to the weld beam can be included with the multi-component model to obtain a better fit of simulation results with experimental measurements. The result of this approach shows that second component of eigenstrain has a strong influence on residual stresses. The best fit of residual stress is obtained when eigenstrain distribution length is set to 70 and $90 \mathrm{~mm}$ in as-welded and heat-treated models respectively as illustrated in Figure 11.

Profile plots of through the thickness averaged results in Figure 12 show good agreement with experimentally determined displacement and residual stress distributions. As it is expected, tensile residual stresses in the weld zone cause formation of compressive residual stresses away from weld zone. However, three-dimensional visual representation of residual 
stress in Figures 19 and 20 shows that calculations of multi-component model fails at out of Plane A.

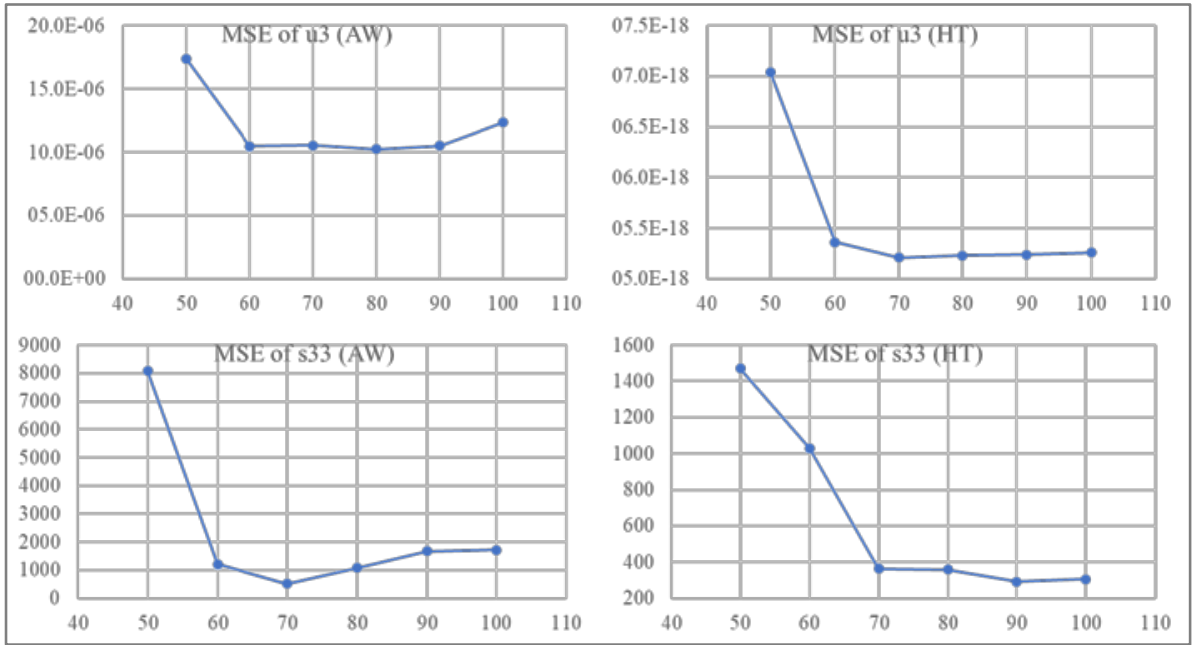

Figure 11. Mean squared error (MSE) of longitudinal displacement (u3) and residual stress (s33) w.r.t eigenstrain distribution width (in $\mathrm{mm}$ ) on the cut surface of as-welded (AW) and heat-treated (HT) samples after multi-component single matrix eigenstrain reconstruction process.

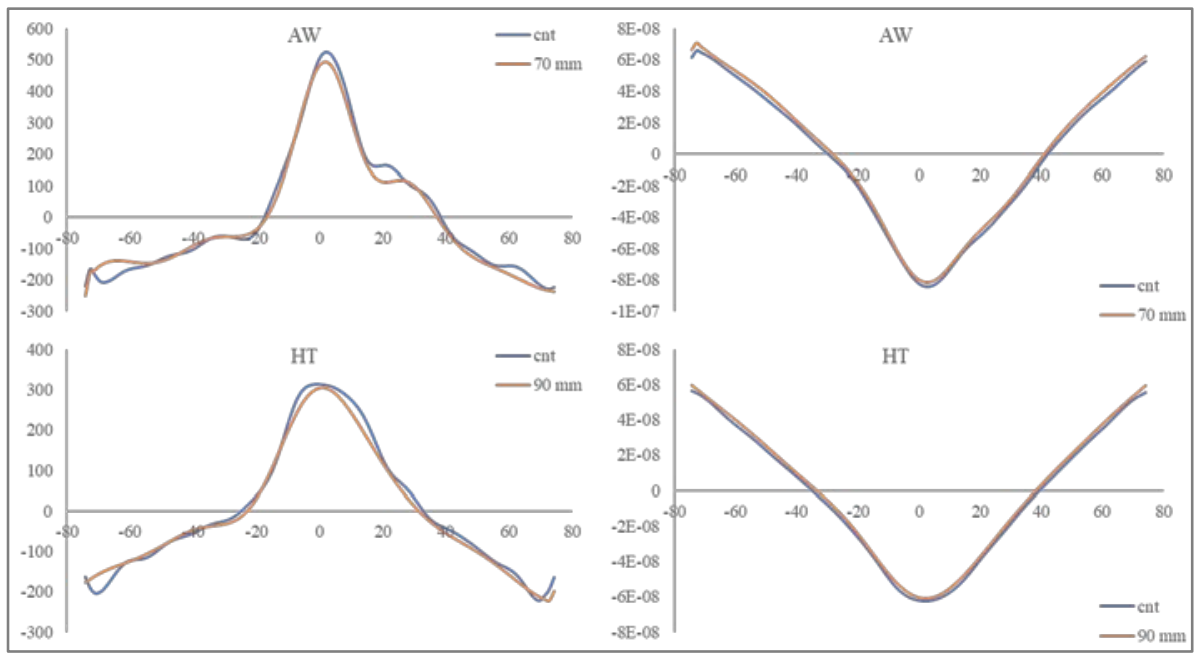

Figure 12. Transversal profile distributions of the through-thickness average of displacement (in $\mathrm{mm}$ ) and longitudinal residual stress (in $\mathrm{MPa}$ ) obtained using multi-component single 
matrix eigenstrain reconstruction process at eigenstrain distribution widths of $70 \mathrm{~mm}$ for the as-welded model and $90 \mathrm{~mm}$ for the heat-treated model.

\subsection{The Multi-Component Iterative Solution}

Limitations of the conventional multi-component model created a requirement for developing a new approach to solve the multi-component eigenstrain problem. For this purpose, a new iterative model is developed based on the assumption that $y y$ and $z z$ components of eigenstrain are identical. Results show that magnitude of residual stress fit increases with increasing distribution length and decreases with a further increase after a best fitting distribution length. The best fitting distribution length for the as-welded model is $80 \mathrm{~mm}$ while it is $100 \mathrm{~mm}$ for heat treated model. As it is expected, eigenstrain distribution length in the heat-treated model is wider than the as-welded model. Figure 13 shows that distribution length has almost no effect on the magnitude of fit in heat treated model after $100 \mathrm{~mm}$.

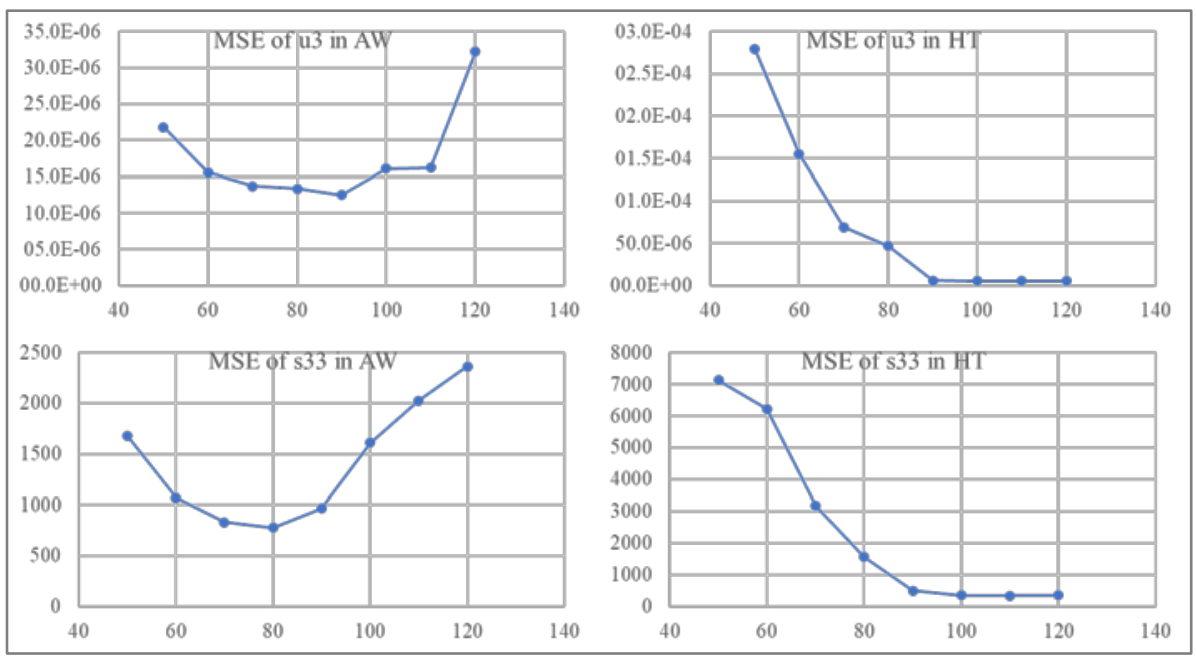

Figure 13. Mean squared error (MSE) of longitudinal displacement (u3) and residual stress (s33) w.r.t eigenstrain distribution width (in $\mathrm{mm}$ ) on the cut surface of as-welded (AW) and heat-treated (HT) samples after iterative eigenstrain reconstruction process. 
Figure 14 illustrates the through-thickness averaged profile plots of the multi-component iterative solution. Displacement and residual stress calculations obtained with this solution process are in good agreement with measured displacement data from CMM measurements and residual stress calculations of contour method. Distributions of $y y$ and zz components of eigenstrain in as-welded and heat-treated models of the multi-component iterative solution are illustrated in Figures 15 and 16. Because of being locked, yy and zz components have the same distribution with different magnitudes in each solution. As it is expected, eigenstrain distribution in the heat-treated model is wider than the as-welded model.

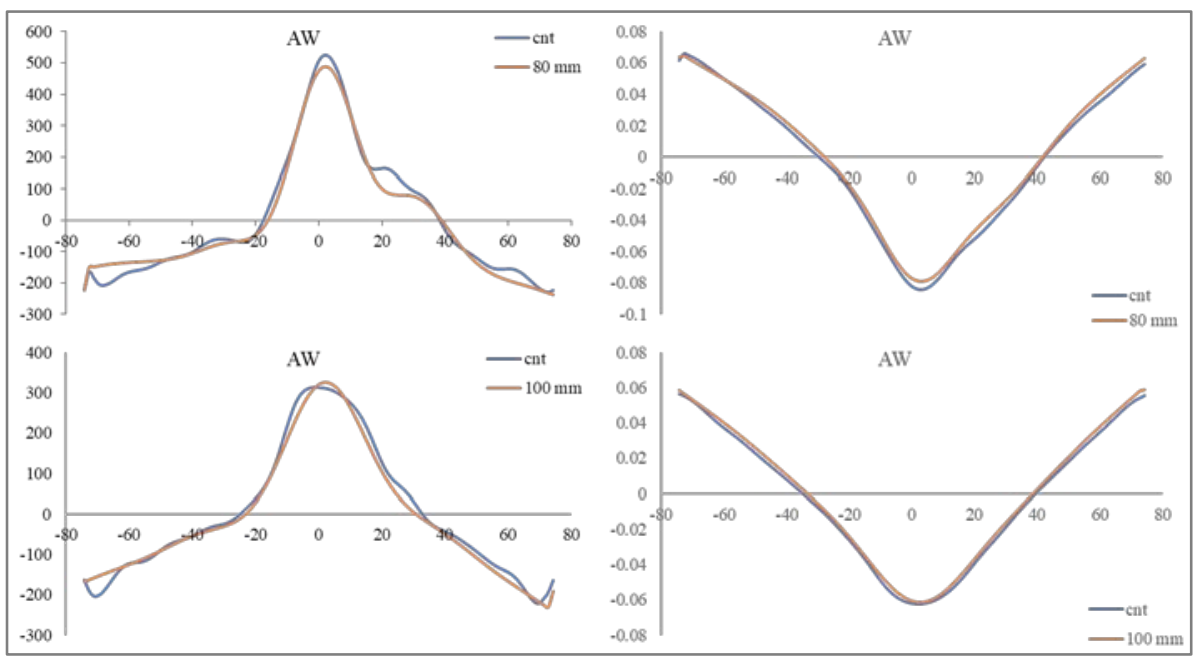

Figure 14. Transversal profile distributions of the through-thickness average of displacement (in $\mathrm{mm}$ ) and longitudinal residual stress (in $\mathrm{MPa}$ ) obtained using multi-component iterative eigenstrain reconstruction process at eigenstrain distribution widths of $80 \mathrm{~mm}$ for the aswelded model and $100 \mathrm{~mm}$ for the heat-treated model. 


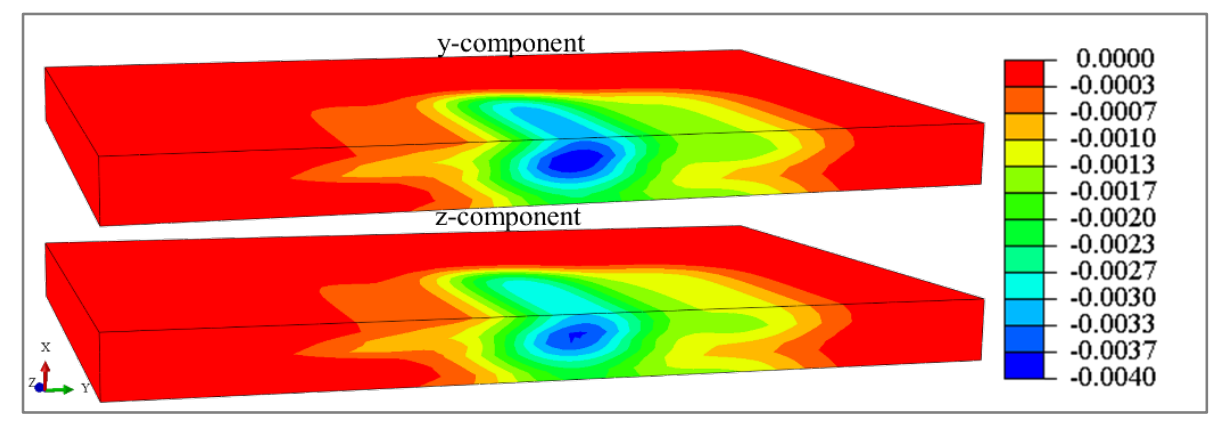

Figure 15. 3D distributions of $\varepsilon_{y y}^{*}$ and $\varepsilon_{z z}^{*}$ in as-welded model, determined using multicomponent iterative eigenstrain reconstruction process.

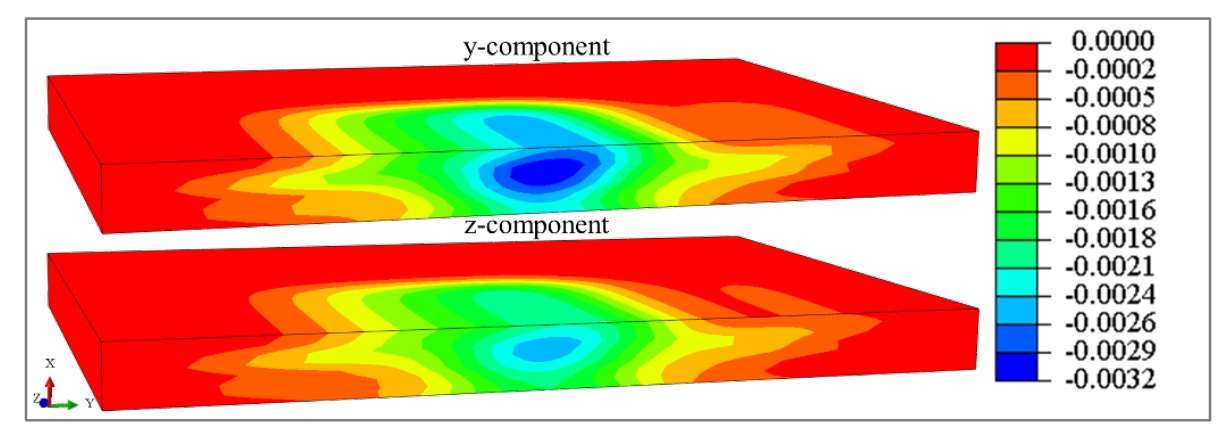

Figure 16. 3D distributions of $\varepsilon_{y y}^{*}$ and $\varepsilon_{z z}^{*}$ in heat-treated model, determined using multicomponent iterative eigenstrain reconstruction process.

\subsection{Comparison of Models and Prediction for the Whole Body}

The model for solving inverse eigenstrain problem is based on fitting of displacements calculated by the finite element simulation with experimentally determined displacement data. This process allows quantification of the quality of the fit in terms of mean squared error. The best eigenstrain distribution size in terms of quantified quality of fit is determined with each model separately. However, the quality of fit on the surface is not sufficient, because, the aim of eigenstrain reconstruction process is to predict residual stress distribution in the whole body. 


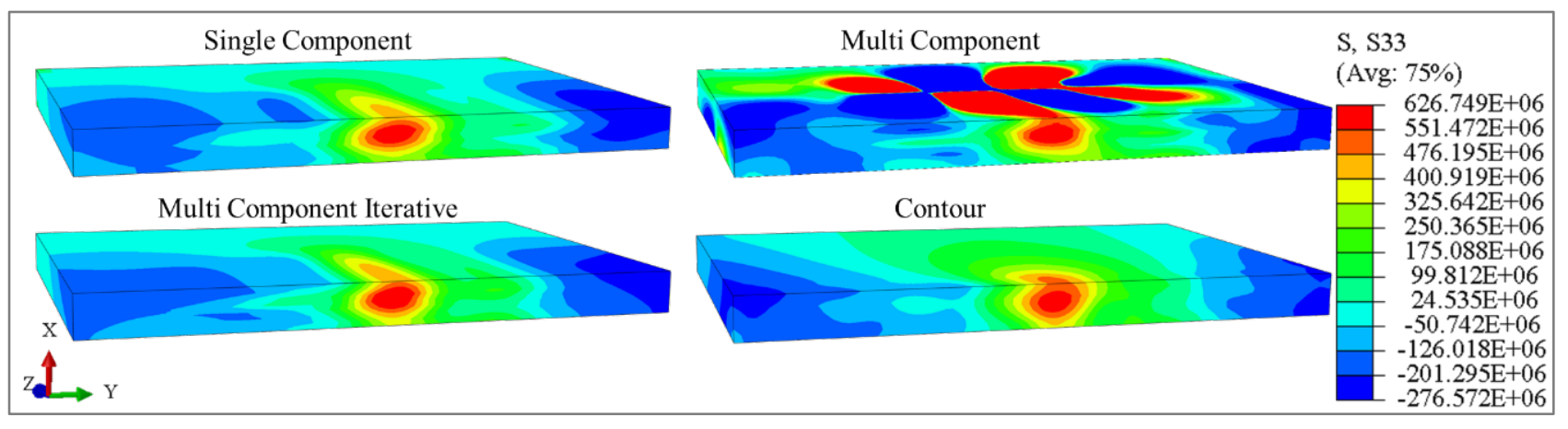

Figure 17. 3D distribution of longitudinal residual stress (in $\mathrm{Pa}$ ) obtained using three eigenstrain reconstruction and contour methods in as-welded model.

Bead on plate specimen design was used in previous studies by the European Network on Neutron Techniques Standardization for Structural Integrity (NeT) for the purpose of investigation of welding residual stress and Smith et al. [25] published a summary of these studies. In those studies, weld bead is laid on the material surface or a slot with finite length is filled with the weld bead. Results of bead-on-plate weld design can be found in the publications of Smith and Smith [26] and Muránsky et al. [27]. These studies provide idea about distribution of different component of residual stress in this type of weld specimen designs. Three-dimensional contour maps of as-welded and heat-treated solutions of all three inverse eigenstrain models are given in Figures 17 and 18. In-volume residual stresses calculated using multi-component solution is far from expected distribution. The lack of experimental data relative to the yy component of eigenstrain causes creation of a delusive eigenstrain field that gives an expected residual stress map on the plane of fit only.

Depending on this fact, further investigations are done using single-component and multicomponent iterative solutions and results show that in-volume residual stress distributions are appropriate for the bead on plate sample design. 


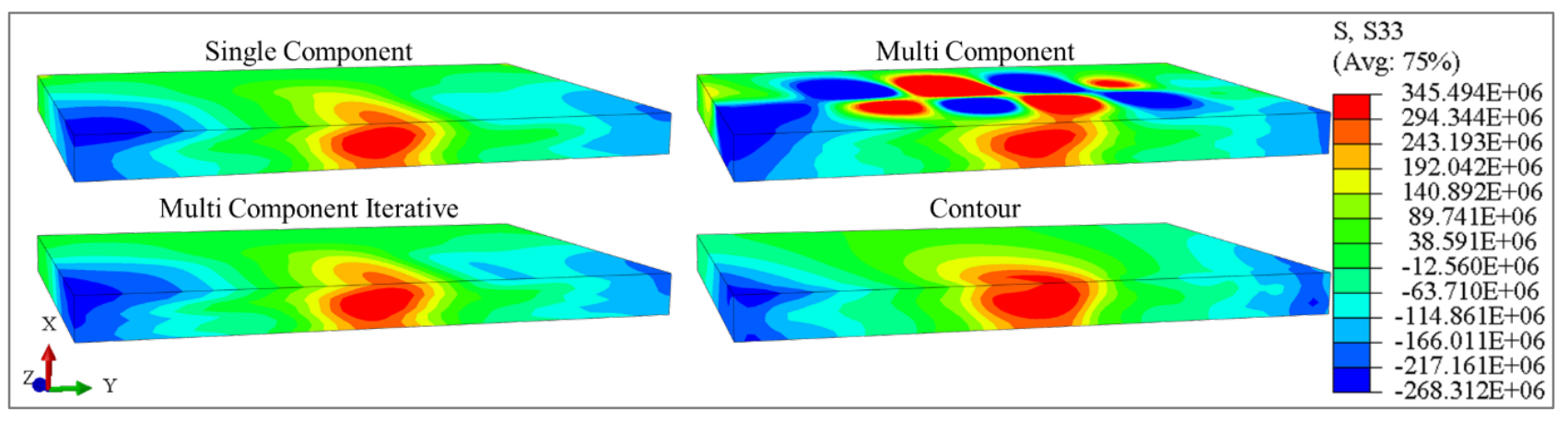

Figure 18. 3D distribution of longitudinal residual stress (in $\mathrm{Pa}$ ) obtained using three eigenstrain reconstruction and contour methods in heat-treated model.

Overall results show that single-component and multi-component iterative models are appropriate for reconstruction of residual stresses in nonuniform weld specimens. Both models provide similar stress maps in the whole body, but single-component model provides these results with a wider eigenstrain distribution range and quality of fit obtained with the single-component solution is lower than the multi-component iterative solution. Figure 19 gives a comparison of the quantified quality of fit of the results with minimum MSE. As a consequence, it can be stated that multi-component iterative solution provides the most reliable result on the determination of permanent plastic strain distribution.

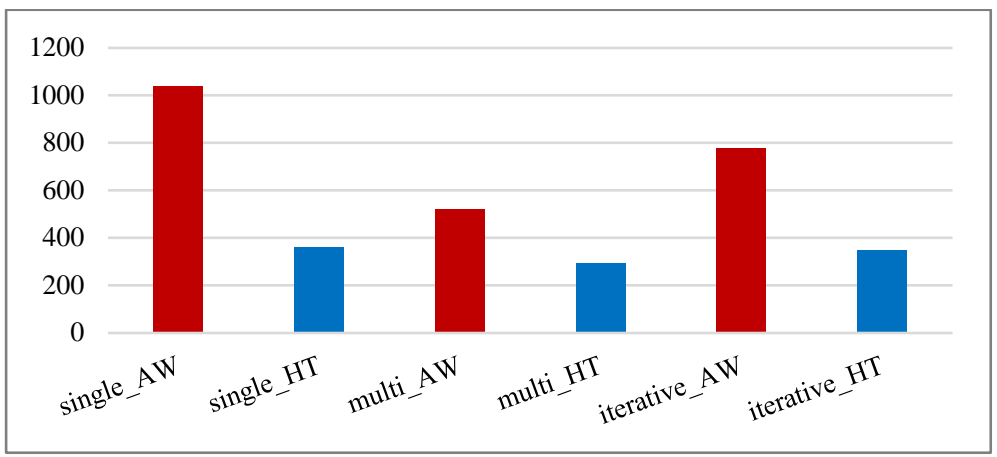

Figure 19. Comparison of quantified quality of fit in terms of MSE of longitudinal residual stress (in $M P a$ ). 
Figure 20 illustrates contour map of variation of longitudinal residual stress distribution in Plane A which is calculated using multi-component iterative model. The influence of beadon-plate specimen design on this distribution can be seen clearly. A study conducted by Ahn et al. [21] using three-dimensional thermal elastic-plastic finite element models of uniform weld designs shows that longitudinal residual stresses appear as tensional stresses around weld beam and transversal distribution stabilizes at around zero stress state. However, longitudinal residual stresses have a balanced state of tensional and compressive longitudinal stresses in the proposed specimen design. This result shows the effect of non-welded part of the specimen on formation and distribution of welding residual stress.

A common result on the distribution of longitudinal residual stress in a plane identical to Plane B is that; tensional longitudinal residual stresses distribute in this zone and magnitude decreases up to zero away from the back end of the weld bead, but it never becomes negative. This distribution is consistent with results of the studies which are performed using bead-onplate specimen design. Examples of these studies can be found in the summary of Smith et. al. [25]. Contour map and profile plots in Figure 22 shows that longitudinal residual stresses in Plane B are consistent with expected results. It must be emphasized that this result is the calculation of multi-component iterative solution process based on the experimental verification of displacement data on Plane A.

The effect of heat treatment process on stress relaxation is presented in Figures 22. Heat treatment reduces the strength of tension stresses in weld zone, but compressive residual stresses keep their magnitude at the edge parts of Plane A, because, the main reason of compressive longitudinal residual stresses along the transversal direction is the non-welded part of the specimen. Heat treatment process reduces the magnitude of permanent plastic 
strains, but, at the same time, this process causes the addition of creep strains that widens the distribution of permanent plastic strains. At the end of the heat-treatment, eigenstrains with extended distribution and reduced magnitude, in the weld zone, causes the formation of compressive stresses, with a similar magnitude of the as-welded specimen, at the edge parts of the body.

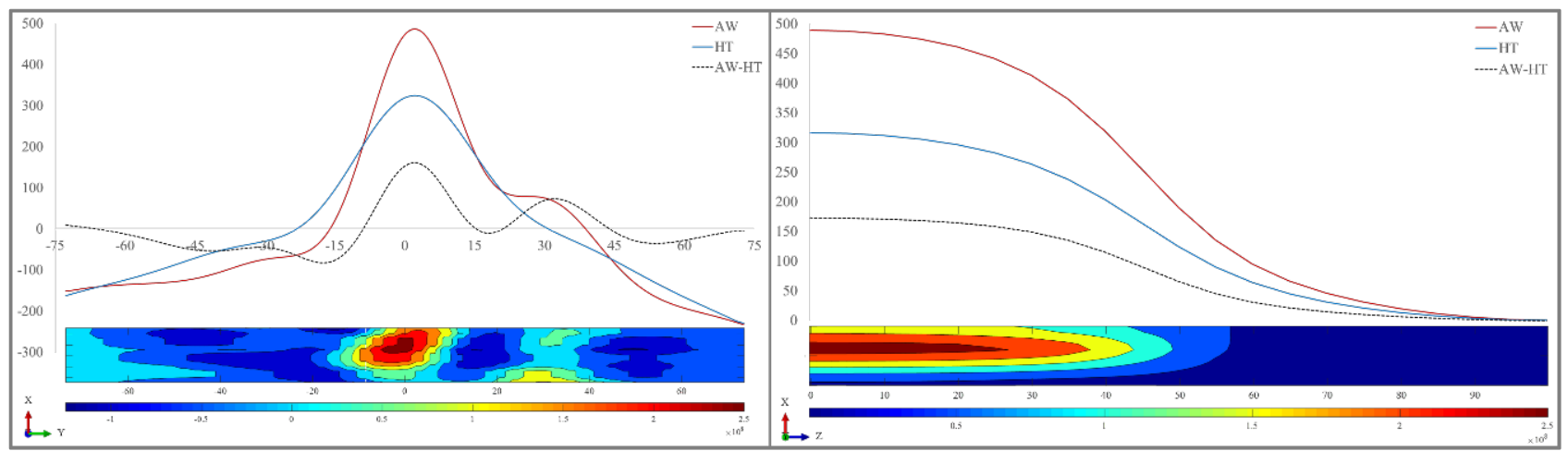

Figure 20. Profile distributions of the through-thickness average of longitudinal residual stress (in $\mathrm{MPa}$ ) obtained using multi-component iterative eigenstrain reconstruction process at eigenstrain distribution widths of $80 \mathrm{~mm}$ for the as-welded (AW) model and $100 \mathrm{~mm}$ for the heat-treated (HT) model and stress reduction (AW-HT) as profile distribution and contour map at Plane A (left) and Plane B (right).

\section{CONCLUSION}

Three different models for inverse eigenstrain problem showed that the main source of welding residual stress is the compressive permanent plastic strains in and around the weld zone and compressive residual stresses, at the edge parts in the transversal direction, are the result of tension stresses formed as a result of these plastic strains. However, the singlecomponent model uses only zz component eigenstrain while the multi-component model is not able to reconstruct residual stresses in a three-dimensional body without using 
information about transversal displacements that distribute within the body. To deal with this problem, investigations focused on the development of a new model based on the assumption that yy and zz components of eigenstrain are identical. Results showed that iterative model provides an improvement on the quality of fit when compared to single-component solution.

The eigenstrain reconstruction process is based on experimentally determined displacement measurements. When the correct eigenstrain field is reconstructed, eigenstrain models calculate residual stresses in the whole body and determine displacements by simulating the cutting process. A large amount of high-resolution displacement data collected by precise CMM measurements allows determination of eigenstrain fields with high accuracy. In addition, this data allows determination of residual stresses with simple elastic calculations and provide highly reliable experimental results. Availability of high-resolution data ensured a highly accurate match between eigenstrain reconstruction process calculations and experimental contour method results.

\section{ACKNOWLEDGEMENT}

This project has received funding from the European Union's Horizon 2020 research and innovation programme under the Marie Sklodowska-Curie grant agreement No 794957 and EPSRC through grant EP/S005072/1 Strategic Partnership in Computational Science for Advanced Simulation and Modelling of Engineering Systems (ASiMoV).

\section{REFERENCES}

1. U.S. Energy Information Agency. International Energy Outlook 2013. Outlook 2013 2013:312. doi:EIA-0484(2013). 
2. Barnes I. Upgrading the Efficiency of the World's Coal Fleet to Reduce CO2 Emissions. IEA Clean Coal Cent 2014:1-99.

3. Furtado HC, Le May I. High Temperature Degradation in Power Plants and Refineries. Mater Res 2004;7:1-103.

4. Withers PJ, Bhadeshia HKDH. Residual stress. Part 2 - Nature and origins. Mater Sci Technol 2001;17:366-75. doi:10.1179/026708301101510087.

5. Fitzpatrick ME, Lodini A. Analysis of Residual Stress by Diffraction Using Neutron and Synchrotron Radiation. vol. 14. 2003. doi:10.1088/0957-0233/14/9/703.

6. Tanala E, Bourse G, Fremiot M, De Belleval JF. Determination of near surface residual stresses on welded joints using ultrasonic methods. NDT E Int 1995;28:83-8. doi:10.1016/0963-8695(94)00013-A.

7. Palanichamy P, Vasudevan M, Jayakumar T. Measurement of residual stresses in austenitic stainless steel weld joints using ultrasonic technique. Sci Technol Weld Join 2009;14:166-71. doi:10.1179/136217108X394753.

8. Javadi Y, Akhlaghi M, Najafabadi MA. Using finite element and ultrasonic method to evaluate welding longitudinal residual stress through the thickness in austenitic stainless steel plates. Mater Des 2013;45:628-42. doi:10.1016/j.matdes.2012.09.038.

9. Uzun F, Bilge AN. Ultrasonic Investigation of the Effect of Carbon Content in Carbon Steels on Bulk Residual Stress. J Nondestruct Eval 2015;34. doi:10.1007/s10921-0150284-x.

10. Prime MB. Cross-Sectional Mapping of Residual Stresses by Measuring the Surface Contour After a Cut. J Eng Mater Technol 2001;123:162. doi:10.1115/1.1345526.

11. Kartal ME, Kang YH, Korsunsky AM, Cocks ACF, Bouchard JP. The influence of welding procedure and plate geometry on residual stresses in thick components. Int J Solids Struct 2016;80:420-9. doi:10.1016/j.ijsolstr.2015.10.001. 
12. Kartal M, Turski M, Johnson G, Fitzpatrick ME, Gungor S, Withers PJ, et al. Residual stress measurements in single and multi-pass groove weld specimens using neutron diffraction and the contour method. Mater Sci Forum 2006;524-525:671-6. doi:10.4028/www.scientific.net/MSF.524-525.671.

13. Prime MB, Gnäupel-Herold T, Baumann JA, Lederich RJ, Bowden DM, Sebring RJ. Residual stress measurements in a thick, dissimilar aluminum alloy friction stir weld. Acta Mater 2006;54:4013-21. doi:10.1016/j.actamat.2006.04.034.

14. Toparli MB, Fitzpatrick ME, Gungor S. Improvement of the Contour Method for Measurement of Near-Surface Residual Stresses from Laser Peening. Exp Mech 2013;53:1705-18. doi:10.1007/s11340-013-9766-x.

15. Traore Y, Paddea S, Bouchard PJ, Gharghouri MA. Measurement of the Residual Stress Tensor in a Compact Tension Weld Specimen. Exp Mech 2013;53:605-18. doi:10.1007/s11340-012-9672-7.

16. Zabeen S, Preuss M, Withers PJ. Residual stresses caused by head-on and $45^{\circ}$ foreign object damage for a laser shock peened Ti-6Al-4V alloy aerofoil. Mater Sci Eng A 2013;560:518-27. doi:10.1016/j.msea.2012.09.097.

17. Carlone P, Citarella R, Sonne MR, Hattel JH. Multiple crack growth prediction in AA2024-T3 friction stir welded joints, including manufacturing effects. Int J Fatigue 2016;90:69-77. doi:10.1016/j.ijfatigue.2016.04.004.

18. Citarella R, Carlone P, Sepe R, Lepore M. DBEM crack propagation in friction stir welded aluminum joints. Adv Eng Softw 2016;101:50-9. doi:10.1016/j.advengsoft.2015.12.002.

19. DeWald AT, Hill MR. Multi-Axial Contour Method for Mapping Residual Stresses in Continuously Processed Bodies. Exp Mech 2006;46:473-90. doi:10.1007/s11340-0068446-5. 
20. Korsunsky AM. A Teaching Essay on Residual Stresses and Eigenstrains. Oxford, United Kingdom: Butterworth-Heinemann; 2017.

21. Ahn J, He E, Chen L, Wimpory RC, Dear JP, Davies CM. Prediction and measurement of residual stresses and distortions in fibre laser welded Ti-6Al-4V considering phase transformation. Mater Des 2017;115:441-57. doi:10.1016/j.matdes.2016.11.078.

22. Mura T. Micromechanics of Defects in Solids. Dordrecht: Netherlands: Kluwer Academic Publishers; 1982.

23. Dong P, Song S, Zhang J. Analysis of residual stress relief mechanisms in post-weld heat treatment. Int J Press Vessel Pip 2014;122:6-14. doi:10.1016/j.ijpvp.2014.06.002.

24. Wang Y, Dong J, Zhang M, Yao Z. Stress relaxation behavior and mechanism of AEREX350 and Waspaloy superalloys. Mater Sci Eng A 2016;678:10-22. doi:10.1016/j.msea.2016.09.077.

25. Smith MC, Smith AC, Wimpory R, Ohms C. A review of the NeT Task Group 1 residual stress measurement and analysis round robin on a single weld bead-on-plate specimen. Int J Press Vessel Pip 2014;120-121:93-140. doi:10.1016/j.ijpvp.2014.05.002.

26. Smith MC, Smith AC. NeT bead-on-plate round robin: Comparison of transient thermal predictions and measurements. Int J Press Vessel Pip 2009;86:96-109. doi:10.1016/j.ijpvp.2008.11.016.

27. Muránsky O, Hamelin CJ, Smith MC, Bendeich PJ, Edwards L. The effect of plasticity theory on predicted residual stress fields in numerical weld analyses. Comput Mater Sci 2012;54:125-34. doi:10.1016/j.commatsci.2011.10.026. 